\title{
Encephalitis associated with cervical lymph nodes tuberculosis in a patient with human immunodeficiency virus infection: A case report
}

\author{
Rizaldy Pinzon ${ }^{1}$ and Hana Kristina ${ }^{1}$ \\ ${ }^{1}$ Duta Wacana Christian University Faculty of Medicine
}

November 1, 2021

\begin{abstract}
The risk of extrapulmonary tuberculosis increases in patients with human immunodeficiency virus (HIV) infections. Encephalitis is a rare form of extrapulmonary tuberculosis infection. We report a case of encephalitis associated with cervical lymph nodes tuberculosis in a patient with human immunodeficiency virus (HIV) infections.
\end{abstract}

Encephalitis associated with cervical lymph nodes tuberculosis in a patient with human immunodeficiency virus infection: A case report

Rizaldy Taslim Pinzon ${ }^{1,2^{*}}$, Hana Kristina ${ }^{1,2}$

1 Duta Wacana Christian University School of Medicine, Yogyakarta, Indonesia

2 Bethesda Hospital, Yogyakarta, Indonesia

* Corresponding author: Rizaldy Taslim Pinzon

Address: Duta Wacana Christian University School of Medicine, Wahidin Sudirohusodo st 5-

25, Yogyakarta 55224, Indonesia. E-mail address: drpinzon17@gmail.com

Key Clinical Message

The risk of extrapulmonary tuberculosis increases in patients with human immunodeficiency virus (HIV) infections. Encephalitis is a rare form of extrapulmonary tuberculosis infection. We report a case of encephalitis associated with cervical lymph nodes tuberculosis in a patient with human immunodeficiency virus (HIV) infections.

\section{Keywords}

Encephalitis, Cervical lymph nodes tuberculosis, Human immunodeficiency virus infection

\section{Introduction}

From 2003 to 2014, 27 countries of the EU/EEA have reported that $83 \%$ of tuberculosis patients had pulmonary infection only and $17 \%$ of tuberculosis patients had extrapulmonary infections ${ }^{1}$. The risk of extrapulmonary tuberculosis increases higher compared with pulmonary tuberculosis in patients with HIV $^{2}$. It is estimated a total of 208,000 deaths from tuberculosis among HIV-positive individuals. In 2019, Indonesia had a tuberculosis burden of $8.5 \%^{3}$.

The most common location of head and neck tuberculosis is cervical lymph nodes ${ }^{4}$. Lymph nodes tuberculosis is common in children and women. Tuberculous cervical lymphadenitis is often found along with the increasing incidence of HIV infection ${ }^{5}$. 
Neurological involvement is reported in $3 \%$ of extrapulmonary tuberculosis infections ${ }^{1}$. Central nervous system tuberculosis usually appears as tuberculous meningitis, tuberculous brain abscess, or intracranial tuberculoma ${ }^{6}$. Its manifestation as encephalopathy is rare and reported more common in children ${ }^{7}$. We report a case of encephalitis associated with cervical lymph nodes tuberculosis in patients with HIV infection.

\section{Case Report}

A 50-year-old man visited the emergency unit with slurred speech, difficulty swallowing, and involuntary movements of the right upper extremity. Prior to complaints of involuntary movement of upper limb, the patient complained of vomiting due to a hot and pressure-like feeling on his throat. Then, he complained of slurred speech and difficulty swallowing. These complaints appeared an hour and a half before arriving at the hospital. All complaints disappeared as soon as he arrived at the hospital. The patient also complained of numbness of the right hand since one month before arrival. However, there were no complaints of fever, headache, or cough. The patient had a history of hospitalization 6 months before with dizziness. His next house neighbour was reported having cough for months but the cause was unknown.

Based on physical examination, the patient was found to be compos mentis. The patient has a temperature of $36.1^{\circ} \mathrm{C}$, pulse of 112 beats/minute, respiratory rate of 22 breaths/minute, blood pressure of $149 / 81 \mathrm{~mm} / \mathrm{Hg}$., and oxygen saturation of $96 \%$. The patient had a fever with a temperature of $38.0^{\circ} \mathrm{C}$ on the first night of hospitalization, then the temperature immediately return to normal after receiving paracetamol. He has no neck stiffness but has bilateral cervical lymphadenitis. Based on neurological examination, numbness was found in the patient's right palm. The result of cranial nerves and motor examinations were normal.

Further, computed tomography (CT) scan of the head showed a hypodense lesion in the left parietal lobe (Fig.1). The chest radiograph showed peribronchial and parenchymal infiltrates (Fig. 2). Laboratory tests showed a positive HIV antibody test. Cervical lymph node biopsy showed the presence of cheese granulomatous inflammation and positive acid-fast bacilli. 


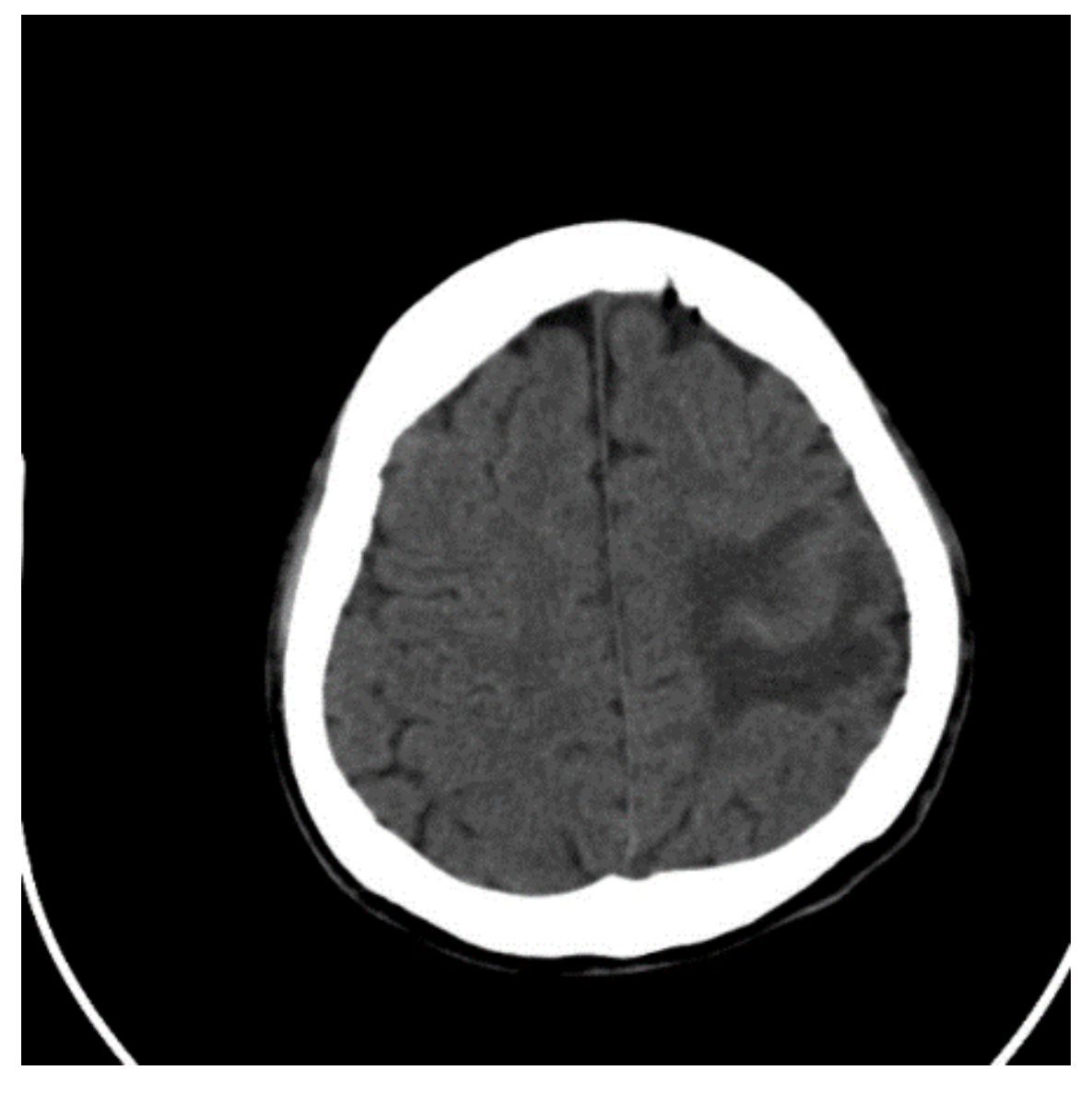

Figure 1. Hypodense lesion in left parietal lobe on CT scan 


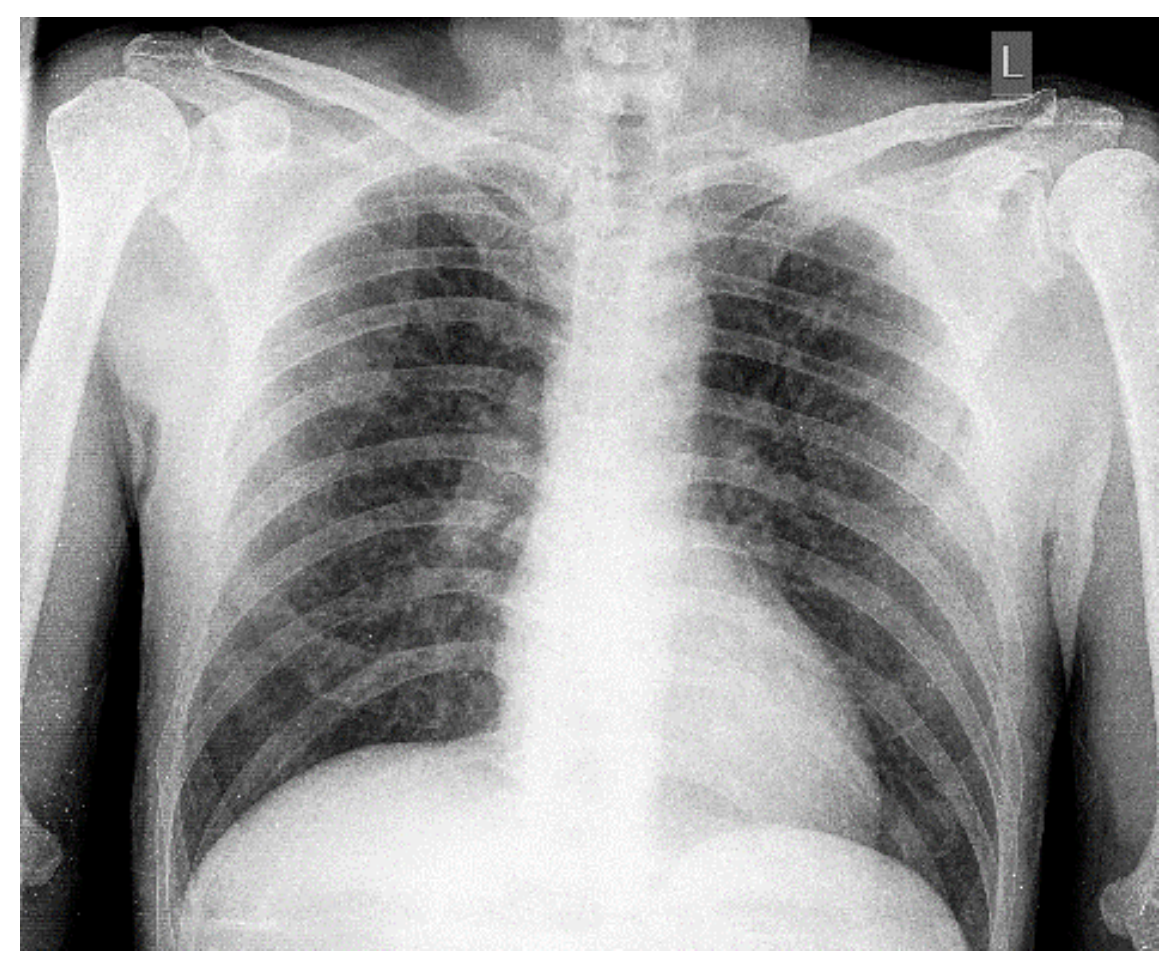

Figure 2. Peribronchial and parenchymal infiltrates on chest X-ray

The patient was given a fixed-dose combination of antituberculosis therapy consisting of isoniazid (225 $\mathrm{mg})$, rifampicin $(450 \mathrm{mg})$, ethambutol $(825 \mathrm{mg})$, and pyrazinamide $(1200 \mathrm{mg})$. The follow up showed significant improvement.

\section{Discussion}

Encephalitis is caused by inflammation of the brain parenchyma causing neurological dysfunction. The major criterion of encephalitis is an altered mental status that remains for more than 24 hours without an alternative diagnosis. Then, there are six minor criteria such as (1) fever $>38^{\circ} \mathrm{C}$ within 72 hours before or after symptom onset, (2) new onset of focal neurologic disorder, (3) abnormality on electroencephalography consistent with encephalitis and not secondary to another etiology, (4) CSF white blood cells count $>5$ $\mathrm{mm}^{2},(5)$ acute onset of neuroimaging abnormality consistent with encephalitis, (6) seizures not associated with a pre-existing seizure disorder. The presence of 2 minor criteria indicates possible encephalitis and the presence of more than 3 minor criteria indicates probable or confirmed encephalitis (if the etiologic agent is confirmed by polymerase chain reaction, serology, or brain biopsy) ${ }^{8}$. This case met 2 minor criteria of fever of $38^{\circ} \mathrm{C} 24$ hours after symptom onset and the presence of new onset of focal neurologic disturbances of dysarthria, dysphagia, and involuntary movements.

Clinical overlap between encephalitis and encephalopathy may occur in which encephalopathy leads to a clinical condition of altered mental status that can manifest as disorientation, confusion, or other cognitive impairment, with or without evidence of brain tissue inflammation. Encephalopathy can be triggered by some toxic or metabolic conditions but sometimes occurs in response to infectious agents such as the influenza virus and Bartonella henselae ${ }^{9,10}$.

Patients with neurotuberculosis have few specific symptoms. Delay in diagnosis must be considered, usually due to a mild initial neurologic presentation in most cases, if compared with other infectious encephalitis ${ }^{11}$. Based on a report in France, only $20 \%$ of patients had a previous history of tuberculosis. Besides, only 
$20 \%$ of patients had respiratory symptoms ${ }^{12}$. In this case, the patient came with a mild initial neurologic presentation and had no respiratory symptoms indicated by no history of cough.

Brain MRI is the best device for the diagnosis of encephalitis. CT scan is used only when MRI is not possible $^{13}$. There are no specific features for encephalitis except in brain abscess or granulomatous lesions cases. A study in France revealed that CT scan and MRI scan were normal in 8 of 17 patients. It means that there is a possibility of a diagnosis of tuberculosis being ruled out in cases of normal imaging ${ }^{12}$.

There are no conventional lymphatics in the brain, but physiological studies showed immunologically significant drainage from the brain to cervical lymph nodes. Perivascular lymphatic drainage from the central nervous system has an important role in neuroimmunological reactions on autoimmune diseases and the same mechanism also plays a role in infection of the central nervous system ${ }^{14-16}$.

Specific antituberculosis therapy can be associated with the prolonged onset of action, especially in patients with severe brain damage ${ }^{17}$. The standard recommended therapy is fixed-dose combinations of 4 drugs (isoniazid, rifampicin, ethambutol, and pyrazinamide) for 6 months intensively. Sudden discontinuation of treatment is an independent risk factor for mortality in patients with central nervous system tuberculosis ${ }^{11}$.

\section{Conclusion}

The HIV risk factor has to be considered in extrapulmonary tuberculosis. The physician has to observe for signs and symptoms that can be atypical of tuberculous encephalitis. Delay in the initiation of antimicrobial therapy in patients with encephalitis tuberculosis is associated with increased mortality and risk of neurological sequelae ${ }^{18}$.

\section{Conflict of Interest}

None declared.

\section{Authorship}

RTP was responsible for the initial manuscript, data processing, imaging report, and supervision. HK was involved in the manuscript's preparation and editing.

\section{Funding}

This research did not receive any specific grant from funding agencies in the public, commercial, or not-forprofit sectors.

\section{References}

1. Sotgiu, G., D. Falzon, V. Hollo, et al. 2017. Determinants of site of tuberculosis disease: An analysis of European surveillance data from 2003 to 2014. PLoS One 12 : 1-14.

2. Shivakoti, R., D. Sharma, G. Mamoon, and K. Pham. 2017. Association of HIV infection with extrapulmonary tuberculosis: a systematic review.Infection 45 : 11-21.

3. World Health Organization. Global Tuberculous Report 2020. Geneva: World Health Organization; 2020.

4. Pang, P., W. Duan, S. Liu, et al. 2018. Clinical study of tuberculosis in the head and neck region - 11 years' experience and a review of the literature article. Emerg. Microbes Infect .7 : 1-10.

5. Rajasekaran, S., M. Gunasekaran, D. D. Jayakumar, D. Jeyaganesh, and V. Bhanumath. 2001. Tuberculosis cervical lymphadenitis in HIV positive and negative patients. indian J. Tuberc . 48 : 201-204.

6. Kim, H. J., K. W. Shim, M. K. Lee, et al. 2011. Tuberculous encephalopathy without meningitis: Pathology and brain MRI findings.Eur. Neurol . 65 : 156-159.

7. Dev, N., M. Bhowmick, S. Chaudhary, and J. Kant. 2019. Tuberculous Encephalopathy without Meningitis: A Rare Manifestation of Disseminated Tuberculosis. Int J Mycobacteriology . 8 : 406-408. 
8. Venkatesan, A., A. R. Tunkel, K. C. Bloch, et al. 2013. Case definitions, diagnostic algorithms, and priorities in encephalitis: Consensus statement of the international encephalitis consortium.Clin. Infect. Dis . 57 : 1114-1128.

9. Tunkel, A. R., C. A. Glaser, K. C. Bloch, et al. 2008. The management of encephalitis: Clinical practice guidelines by the Infectious Diseases Society of America. Clin. Infect. Dis . $47: 303-327$.

10. Weitkamp, J. H., M. D. Spring, T. Brogan, H. Moses, K. C. Bloch, and P. F. Wright. 2004. Influenza A virus-associated acute necrotizing encephalopathy in the United States. Pediatr Infect. Dis. J .23 : 259-263.

11. Stahl, J. P. 2019. Tuberculous Encephalitis. Pp. 121-130 in A Sener, and H. Erdem. Extrapulmonary Tuberculosis . Basel: Springer Nature, 2019

12. Honnorat, E., T. De Broucker, A. Mailles, and J. P. Stahl. 2013. Encephalitis due to Mycobacterium tuberculosis in France. Med. Mal. Infect . 43 : 230-238.

13. Stahl, J. P., P. Azouvi, F. Bruneel, et al. 2017. Guidelines on the management of infectious encephalitis in adults. Med. Mal. Infect . 47: 179-194.

14. Weller, R. O., E. Djuanda, H. Y. Yow, and R. O. Carare. 2009. Lymphatic drainage of the brain and the pathophysiology of neurological disease. Acta Neuropathol . 117 : 1-14.

15. van Zwam, M., R. Huizinga, N. Heijmans, et al. 2008. Surgical excision of CNS-draining lymph nodes reduces relapse severity in chronic-relapsing experimental autoimmune encephalomyelitis. J. Pathol. 217 : 543-551.

16. Thwaites, G. E., R. van Toorn, and J. Schoeman. 2013. Tuberculous meningitis: More questions, still too few answers. Lancet Neurol .12 : 999-1010.

17. Goulenok, T., R. Buzelé, X. Duval, F. Bruneel, J.P. Stahl, and B. Fantin. 2017. Management of adult infectious encephalitis in metropolitan France. Med. Mal. Infect . 47 : 206-220. 

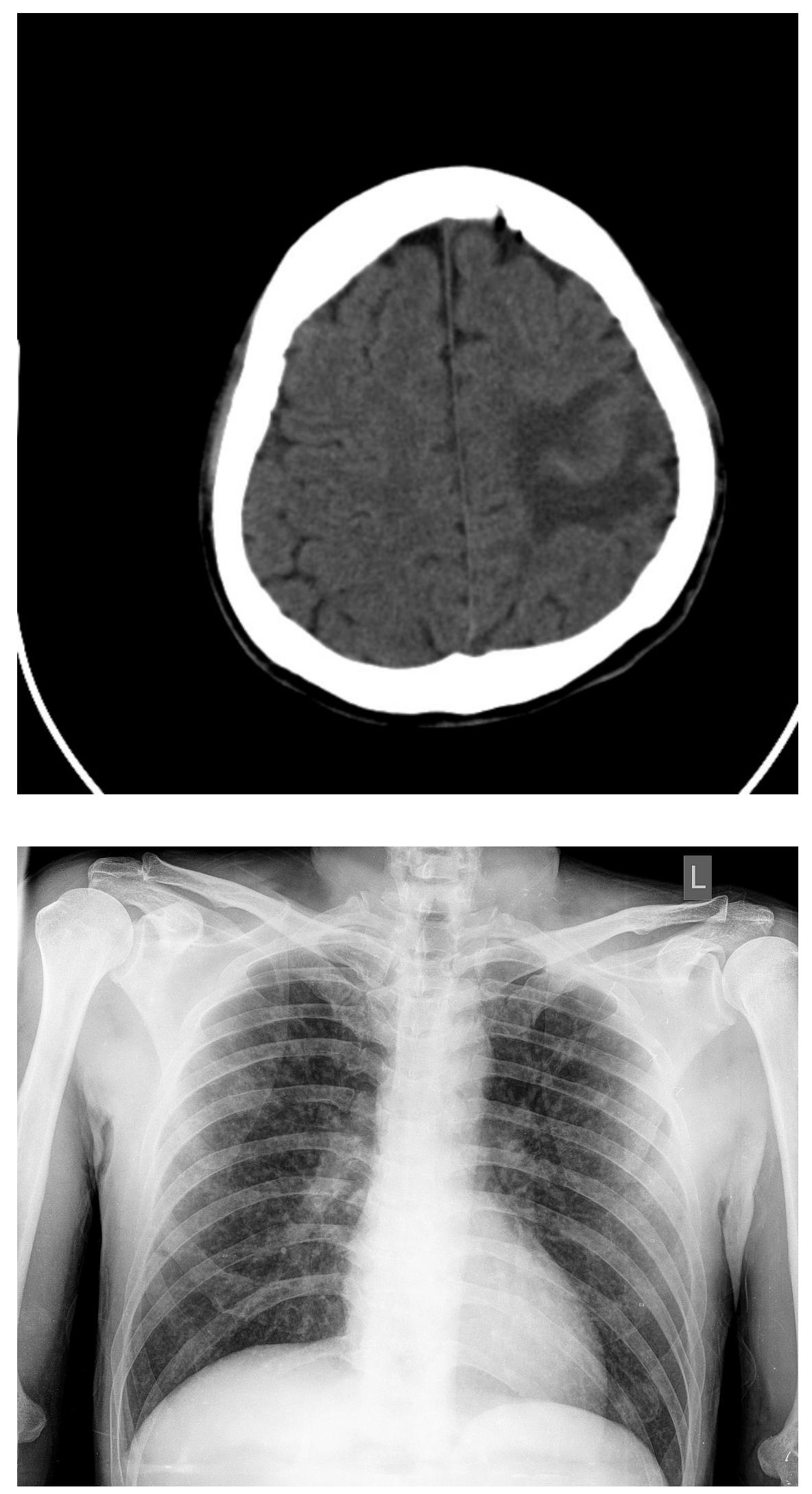